\title{
DESIGN OF A DIGITAL REACTION-DIFFUSION SYSTEM FOR RESTORING BLURRED FINGERPRINT IMAGES
}

\author{
Koichi Ito, Takafumi Aoki and Tatsuo Higuchi \\ Graduate School of Information Sciences, Tohoku University \\ Aoba-yama 05, Sendai, 980-8579 JAPAN \\ Phone: +81-22-217-7169, Fax: +81-22-263-9308, \\ E-mail: ito@aoki.ecei.tohoku.ac.jp
}

\begin{abstract}
This paper presents an algorithm for fingerprint image restoration using a Digital Reaction-Diffusion System (DRDS). The DRDS is a model of a discrete-time discrete-space nonlinear reactiondiffusion dynamical system, which is useful for generating biological textures, patterns and structures. This paper focuses on design and evaluation of a special DRDS having a capability of restoring incomplete fingerprint images. The phase-only image matching technique is employed to evaluate the similarity between the original fingerprint images and the restored images. The proposed algorithm is useful for person identification applications using fingerprint images.
\end{abstract}

\section{INTRODUCTION}

Living organisms can create a remarkable variety of patterns and forms from genetic information. In embryology, the development of patterns and forms is sometimes called Morphogenesis. In 1952, Alan Turing suggested that a system of chemical substances, called morphogens, reacting together and diffusing through a tissue, is adequate to account for the main phenomena of morphogenesis [1]. From an engineering viewpoint, the insights into morphogenesis provide important concepts for devising a new class of intelligent signal processing algorithms inspired by biological pattern formation phenomena [2].

Recently, we have proposed a framework of Digital ReactionDiffusion System (DRDS) - a discrete-time discrete-space reactiondiffusion dynamical system - for designing signal processing models exhibiting active pattern/texture formation capability [3]. This paper describes an application of the DRDS to fingerprint image restoration. The problem considered here is to restore the original fingerprint patterns from blurred fingerprint images. We design a special reaction-diffusion system to generate the most likely fingerprint pattern for a given incomplete fingerprint image. The proposed system is useful for identifying a person even from a blurred fingerprint image and could enhance the performance of conventional fingerprint identification systems. The restoration capability is evaluated by using the phase-only matching technique [4] for fingerprint identification, which has already been applied to practical fingerprint identification systems by the authors' group [5].

\section{DIGITAL REACTION-DIFFUSION SYSTEM}

A Digital Reaction-Diffusion System (DRDS) - a model of a discretetime discrete-space reaction-diffusion dynamical system - can be naturally derived from the original reaction-diffusion system defined in continuous space and time. The general $M$-morphogen reaction-diffusion system with two-dimensional (2-D) space indices $\left(r_{1}, r_{2}\right)$ is written as

$$
\frac{\partial \tilde{\boldsymbol{x}}\left(t, r_{1}, r_{2}\right)}{\partial t}=\tilde{\boldsymbol{R}}\left(\tilde{\boldsymbol{x}}\left(t, r_{1}, r_{2}\right)\right)+\tilde{\boldsymbol{D}} \nabla^{2} \tilde{\boldsymbol{x}}\left(t, r_{1}, r_{2}\right)
$$

where

$$
\begin{aligned}
& \tilde{\boldsymbol{x}}=\left[\tilde{x}_{1}, \tilde{x}_{2}, \cdots, \tilde{x}_{M}\right]^{T}, \\
& \tilde{x}_{i}: \text { concentration of the } i \text {-th morphogen, } \\
& \tilde{\boldsymbol{R}}(\tilde{\boldsymbol{x}})=\left[\tilde{R}_{1}(\tilde{\boldsymbol{x}}), \tilde{R}_{2}(\tilde{\boldsymbol{x}}), \cdots, \tilde{R}_{M}(\tilde{\boldsymbol{x}})\right]^{T}, \\
& \tilde{R}_{i}(\tilde{\boldsymbol{x}}): \text { reaction kinetics for the } i \text {-th morphogen, } \\
& \tilde{\boldsymbol{D}}=\operatorname{diag}\left[\tilde{D}_{1}, \tilde{D}_{2}, \cdots, \tilde{D}_{M}\right], \\
& \quad \operatorname{diag} \text { : diagonal matrix, } \\
& \tilde{D}_{i}: \text { diffusion coefficient of the } i \text {-th morphogen. }
\end{aligned}
$$

We now sample a continuous variable $\tilde{\boldsymbol{x}}$ in (1) at the time sampling interval $T_{0}$, and at the space sampling intervals $T_{1}$ and $T_{2}$. Assuming discrete time-index to be given by $n_{0}$ and discrete space indices to be given by $\left(n_{1}, n_{2}\right)$, we have

$$
\boldsymbol{x}\left(n_{0}, n_{1}, n_{2}\right)=\tilde{\boldsymbol{x}}\left(n_{0} T_{0}, n_{1} T_{1}, n_{2} T_{2}\right) .
$$

Using this discritization, the general DRDS can be obtained as

$$
\begin{aligned}
\boldsymbol{x}\left(n_{0}+1, n_{1}, n_{2}\right)=\boldsymbol{x}\left(n_{0}, n_{1}, n_{2}\right) \\
\quad+\boldsymbol{R}\left(\boldsymbol{x}\left(n_{0}, n_{1}, n_{2}\right)\right)+\boldsymbol{D}(l * \boldsymbol{x})\left(n_{0}, n_{1}, n_{2}\right),
\end{aligned}
$$

where

$$
\begin{aligned}
& \boldsymbol{x}=\left[x_{1}, x_{2}, \cdots, x_{M}\right]^{T}, \\
& \boldsymbol{R}=T_{0} \tilde{\boldsymbol{R}}=\left[R_{1}(\boldsymbol{x}), R_{2}(\boldsymbol{x}), \cdots, R_{M}(\boldsymbol{x})\right]^{T}, \\
& \boldsymbol{D}=T_{0} \tilde{\boldsymbol{D}}=\operatorname{diag}\left[D_{1}, D_{2}, \cdots, D_{M}\right], \\
& l\left(n_{1}, n_{2}\right)= \begin{cases}\frac{1}{T_{1}^{2}} & \left(n_{1}, n_{2}\right)=(-1,0),(1,0) \\
\frac{1}{T_{2}^{2}} & \left(n_{1}, n_{2}\right)=(0,-1),(0,1) \\
-2\left(\frac{1}{T_{1}^{2}}+\frac{1}{T_{2}^{2}}\right) & \left(n_{1}, n_{2}\right)=(0,0) \\
0 & \text { otherwise, }\end{cases}
\end{aligned}
$$




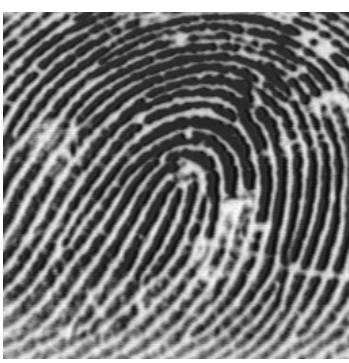

(a)

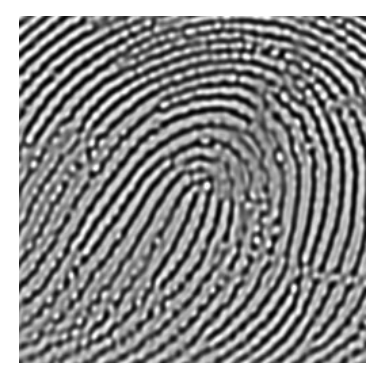

(b)
Figure 1: Enhancement of a fingerprint image: (a) original image, (b) enhanced image.

and $*$ is the spatial convolution operator defined as

$$
\begin{aligned}
(l * \boldsymbol{x})\left(n_{0}, n_{1}, n_{2}\right) & {\left[\begin{array}{c}
\left(l * x_{1}\right)\left(n_{0}, n_{1}, n_{2}\right) \\
\left(l * x_{2}\right)\left(n_{0}, n_{1}, n_{2}\right) \\
\vdots \\
\left(l * x_{M}\right)\left(n_{0}, n_{1}, n_{2}\right)
\end{array}\right] } \\
= & {\left[\begin{array}{c}
\sum_{p_{1}=-1}^{1} \sum_{p_{2}=-1}^{1} l\left(p_{1}, p_{2}\right) x_{1}\left(n_{0}, n_{1}-p_{1}, n_{2}-p_{2}\right) \\
\sum_{p_{1}=-1}^{1} \sum_{p_{2}=-1}^{1} l\left(p_{1}, p_{2}\right) x_{2}\left(n_{0}, n_{1}-p_{1}, n_{2}-p_{2}\right) \\
\sum_{p_{1}=-1}^{1} \sum_{p_{2}=-1}^{1} l\left(p_{1}, p_{2}\right) x_{M}\left(n_{0}, n_{1}-p_{1}, n_{2}-p_{2}\right)
\end{array}\right] . }
\end{aligned}
$$

In this paper, we use the two-morphogen DRDS $(M=2)$ with the Brusselator reaction kinetics, which is one of the most widely studied chemical oscillators [6]. The two-morphogen Brusselatorbased DRDS is defined as follows:

$$
\begin{aligned}
& {\left[\begin{array}{l}
x_{1}\left(n_{0}+1, n_{1}, n_{2}\right) \\
x_{2}\left(n_{0}+1, n_{1}, n_{2}\right)
\end{array}\right]=\left[\begin{array}{l}
x_{1}\left(n_{0}, n_{1}, n_{2}\right) \\
x_{2}\left(n_{0}, n_{1}, n_{2}\right)
\end{array}\right]} \\
& +\quad\left[\begin{array}{c}
R_{1}\left(x_{1}\left(n_{0}, n_{1}, n_{2}\right), x_{2}\left(n_{0}, n_{1}, n_{2}\right)\right) \\
R_{2}\left(x_{1}\left(n_{0}, n_{1}, n_{2}\right), x_{2}\left(n_{0}, n_{1}, n_{2}\right)\right)
\end{array}\right] \\
& +\quad\left[\begin{array}{c}
D_{1}\left(l * x_{1}\right)\left(n_{0}, n_{1}, n_{2}\right) \\
D_{2}\left(l * x_{2}\right)\left(n_{0}, n_{1}, n_{2}\right)
\end{array}\right],
\end{aligned}
$$

where

$$
\begin{aligned}
& R_{1}\left(x_{1}, x_{2}\right)=T_{0}\left\{k_{1}-\left(k_{2}+1\right) x_{1}+x_{1}^{2} x_{2}\right\} \\
& R_{2}\left(x_{1}, x_{2}\right)=T_{0}\left(k_{2} x_{1}-x_{1}^{2} x_{2}\right) .
\end{aligned}
$$

In this paper, we employ the parameter set: $k_{1}=2, k_{2}=4$, $T_{0}=0.01, D_{1}=T_{0}$ and $D_{2}=5 T_{0}$.

The DRDS thus defined can be used to enhance fingerprint patterns [3]. To do this, we first set the initial fingerprint image in $x_{1}\left(0, n_{1}, n_{2}\right)$, at time 0 . Note that spatial sampling parameters $T_{1}$ and $T_{2}$ should be adjusted according to the inherent spatial frequency of the given fingerprint image. The dynamics (4) has the equilibrium $\left(x_{1}, x_{2}\right)=(2,2)$, and the variation ranges of variables $\left(x_{1}, x_{2}\right)$ are bounded around the equilibrium point as $1 \leq x_{1} \leq 3$ and $1 \leq x_{2} \leq 3$ in the case of given parameter set. Hence, we first scale the $[0,255]$ gray-scale fingerprint image into [1,3] range. The scaled image becomes the initial input $x_{1}\left(0, n_{1}, n_{2}\right)$, while the initial condition of the second morphogen is given by $x_{2}\left(0, n_{1}, n_{2}\right)=2$ (equilibrium). The zeroflux Neumann boundary condition is employed for computing the dynamics. After $n_{0}$ steps of the DRDS computation, we obtain $x_{1}\left(n_{0}, n_{1}, n_{2}\right)$ as the output image, which is scaled back into the $[0,255]$ gray-scale image to produce the final output. Figure 1 shows the enhancement of a fingerprint image using the DRDS.

Our initial observation, however, shows that the DRDS with a spatially isotropic diffusion term (4) often produces some broken ridge lines in processing fingerprint images as shown in Fig. 1(b), since it does not take account of the local orientation of ridge flow. In order to solve this problem, the next section defines an adaptive $D R D S$ model, in which we can use the local orientation of the ridge flow in a fingerprint image to guide the action of DRDS. This can be realized by introducing orientation masks to be convolved with the diffusion terms in DRDS (4).

\section{ADAPTIVE DRDS FOR FINGERPRINT RESTORATION}

In this section, we modify the definition of the simple two-morphogen DRDS (4) to have an adaptive DRDS dedicated to fingerprint restoration tasks. The two-morphogen adaptive DRDS with the Brusselator reaction kinetics can be written as

$$
\begin{aligned}
& {\left[\begin{array}{c}
x_{1}\left(n_{0}+1, n_{1}, n_{2}\right) \\
x_{2}\left(n_{0}+1, n_{1}, n_{2}\right)
\end{array}\right]=\left[\begin{array}{c}
x_{1}\left(n_{0}, n_{1}, n_{2}\right) \\
x_{2}\left(n_{0}, n_{1}, n_{2}\right)
\end{array}\right]} \\
& +\quad\left[\begin{array}{c}
R_{1}\left(x_{1}\left(n_{0}, n_{1}, n_{2}\right), x_{2}\left(n_{0}, n_{1}, n_{2}\right)\right) \\
R_{2}\left(x_{1}\left(n_{0}, n_{1}, n_{2}\right), x_{2}\left(n_{0}, n_{1}, n_{2}\right)\right)
\end{array}\right] \\
& +\quad\left[\begin{array}{c}
D_{1}\left(h_{1}^{n_{1} n_{2}} * l * x_{1}\right)\left(n_{0}, n_{1}, n_{2}\right) \\
D_{2}\left(h_{2}^{n_{1} n_{2}} * l * x_{2}\right)\left(n_{0}, n_{1}, n_{2}\right)
\end{array}\right],
\end{aligned}
$$

where

$$
\begin{gathered}
h_{i}^{m_{1} m_{2}}\left(n_{1}, n_{2}\right): \text { orientation mask at the pixel } \\
\quad\left(m_{1}, m_{2}\right) \text { for the } i \text {-th morphogen, } \\
R_{1}\left(x_{1}, x_{2}\right), R_{2}\left(x_{1}, x_{2}\right): \\
\text { the Brusselator reaction kinetics. }
\end{gathered}
$$

In the above equation, we define the orientation mask $h_{1}^{m_{1} m_{2}}\left(n_{1}, n_{2}\right)$ at the pixel $\left(m_{1}, m_{2}\right)$ as a $32 \times 32$ matrix of real coefficients within the window $\left(n_{1}, n_{2}\right)=(-16,-16) \sim(15,15)$. The mask $h_{1}^{m_{1} m_{2}}\left(n_{1}, n_{2}\right)$ controls the dominant orientation of the generated pattern at every pixel $\left(m_{1}, m_{2}\right)$ according to the local ridge flow in the given fingerprint image. The orientation mask can be automatically derived as follows (Fig. 2): (i) take the Fourier transform of the local image around the pixel $\left(m_{1}, m_{2}\right)$, (ii) extract the dominant ridge orientation $\theta$ from the transformed image, (iii) generate a mask pattern $H_{1}^{m_{1} m_{2}}\left(j \omega_{1}, j \omega_{2}\right)$ having the orientation $\theta$ in frequency domain as

$H_{1}^{m_{1} m_{2}}\left(j \omega_{1}, j \omega_{2}\right)= \begin{cases}1 & \begin{array}{l}\text { for unstable frequency band } \\ \text { (black pixels in Fig. 2(iii) })\end{array} \\ 2 & \text { otherwise }\end{cases}$ 
Local Image

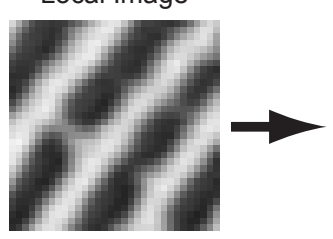

Fourier transform (i)

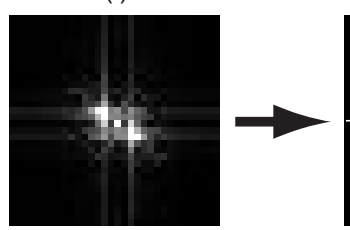

Extract the dominant orientation $\theta$ (ii)

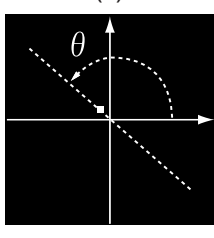

Generate the orientation mask in frequency domain

(iii)

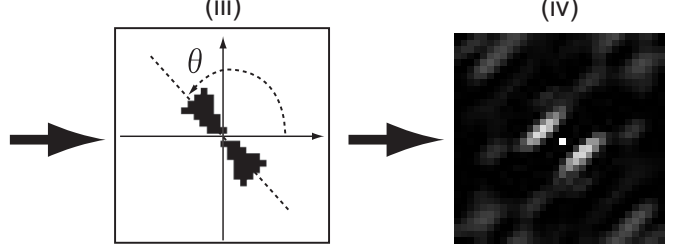

Figure 2: Generation of the orientation mask.

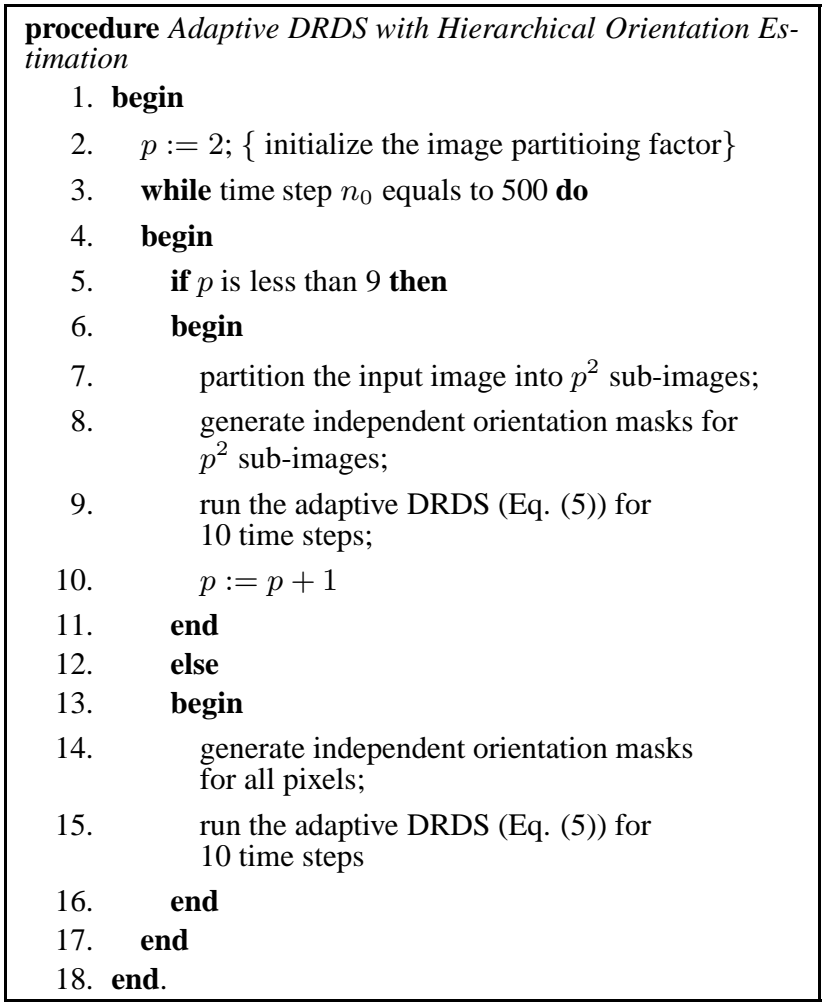

Figure 3: Algorithm for the adaptive DRDS with hierarchical orientation estimation.

and (iv) take the inverse Fourier transform to obtain the orientation mask $h_{1}^{m_{1} m_{2}}\left(n_{1}, n_{2}\right)$. The orientation mask $h_{2}^{m_{1} m_{2}}\left(n_{1}, n_{2}\right)$ for the second morphogen, on the other hand, has the value 1 at the center $\left(n_{1}, n_{2}\right)=(0,0)$, and equals to 0 for the other coordinates $\left(n_{1}, n_{2}\right)$. Thus, the dynamics for the morphogen $x_{2}\left(n_{0}, n_{1}, n_{2}\right)$ does not take account of the local orientation.

In practical situation, it is difficult to obtain the exact orientation masks from blurred fingerprints directly. Addressing this problem, we estimates local orientation masks recursively using a coarse-to-fine approach as shown in Fig. 3. This restoration algorithm starts with rough estimation of local orientation for four sub-images $(p=2)$. The image partitioning factor $p$ gradually in- creases as restoration step $n_{0}$ increases. We can obtain pixel-wise orientation masks $\boldsymbol{h}^{m_{1} m_{2}}\left(n_{1}, n_{2}\right)$ after 80 time steps. This simple strategy makes possible significant improvement in the precision of orientation estimation.

\section{EXPERIMENT}

This section describes a set of experiments for evaluating restoration performance of the proposed algorithm. The problem considered here is to restore the original fingerprint image from its "subsampled" image. For this purpose, we generate a subsampled fingerprint image from the original image as follows: (i) partition the original image into $R \times S$-pixel rectangular blocks, and (ii) select one pixel randomly from every block and eliminate all the other pixels (set 127, middle gray-level, to the pixels). The image thus obtained has the same size as the original image, but the number of effective pixels is reduced to $1 /(R \times S)$.

The restoration capability of the proposed algorithm is evaluated by calculating the similarity between the original fingerprint image and the restored image. To measure the similarity, we employ the phase-only image matching technique [4], which has been proved to have an efficient discrimination capability in practical fingerprint identification tasks [5]. In this experiment, we use 15 distinct fingerprint images (Finger01-Finger15). Restoration experiments are carried out for various subsampling rates $1 /(3 \times 3)$, $1 /(3 \times 4), 1 /(4 \times 4), 1 /(4 \times 5), 1 /(5 \times 5), 1 /(5 \times 6), 1 /(6 \times 6)$, $1 /(6 \times 7), 1 /(7 \times 7), 1 /(7 \times 8)$ and $1 /(8 \times 8)$.

For example, Fig. 4 shows the original image, the subsampled image $\left(n_{0}=0\right)$ and restored images at $n_{0}=100,200$ and 400, respectively, for the case of $1 /(6 \times 6)$ subsampling. We can observe that the fingerprint pattern is reconstructed from the subsampled image gradually as time step $n_{0}$ increases. Figure 5 shows the variation of matching scores calculated between the original image of Finger01 and the restored images of Finger01-Finger15 for the case of subsampling rate $1 /(6 \times 6)$. The matching score of the restored image of Finger01 increases selectively as the number of steps $n_{0}$ increases. For every experimental trial, the optimal discrimination capability could be obtained at around $n_{0}=400$ steps, which is indicated with a vertical dashed line in Fig. 5. The horizontal dashed line indicates the threshold for discrimination.

Table 1 shows the success rate of fingerprint identification for various subsampling rates. In the case where subsampling rates are from $1 /(3 \times 3)$ to $1 /(6 \times 6)$, we can restore the subsampled images completely. This experiment demonstrates a potential ca- 

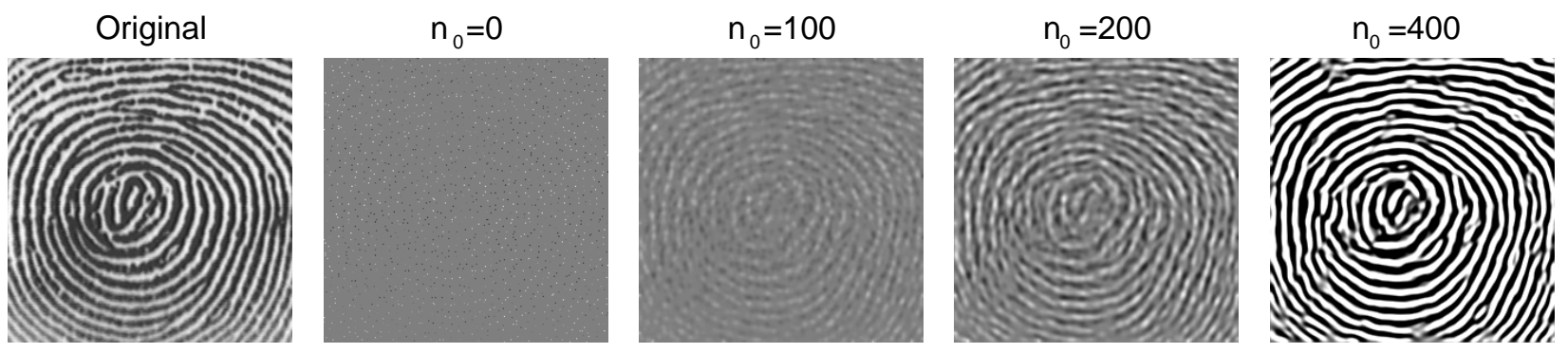

Figure 4: Fingerprint restoration from a subsampled image of Finger01 with subsampling rate $1 /(6 \times 6)$.

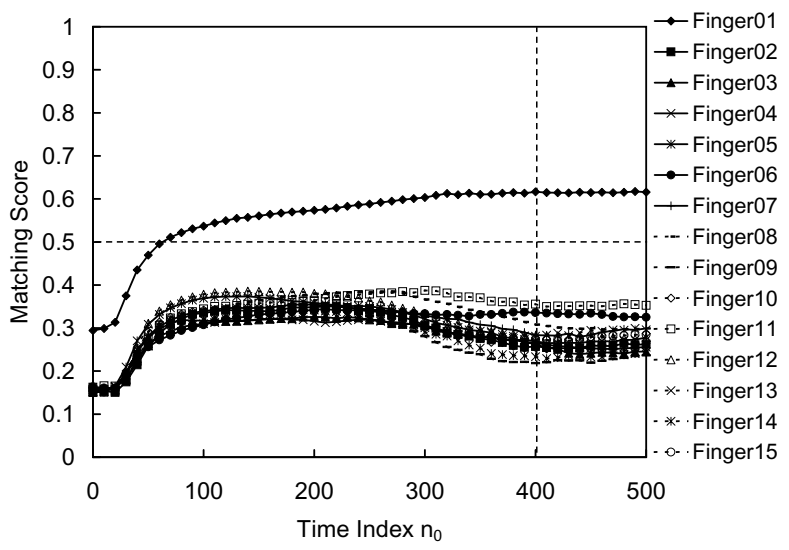

Figure 5: Matching scores between the original image of Finger01 and the restored images of Finger01-Finger15 (restoration from $1 /(6 \times 6)$ subsampled images).

pability of adaptive DRDS to enhance the performance of matching algorithms for blurred fingerprint images. For subsampling rates higher than $1 /(6 \times 6)$, it becomes increasingly difficult to find correct orientation masks. In this region, dedicated fingerprint models (such as deformable templates) may be required for further improvement of restoration performance.

\section{CONCLUSION}

This paper presents an application of the DRDS to fingerprint image restoration. The adaptive DRDS combined with a coarse-tofine orientation estimation technique can reconstruct complete fingerprint patterns even from $1 /(6 \times 6)$-subsampled images. The proposed algorithm may be useful in many person identification applications based on fingerprint images.

\section{REFERENCES}

[1] A. M. Turing, "The chemical basis of morphogenesis," Phil. Trans. R. Soc. London, Vol. B237, pp. 37-72, Aug. 1952.

[2] A. S. Sherstinsky and R. W. Picard,"M-Lattice: From morpho-
Table 1: Identification rate.

\begin{tabular}{|c||c|c|}
\hline $\begin{array}{c}\text { Subsampling } \\
\text { Rate }\end{array}$ & $\begin{array}{c}\text { Number of } \\
\text { Identified Samples }\end{array}$ & $\begin{array}{c}\text { Identification } \\
\text { Rate }\end{array}$ \\
\hline \hline $1 /(3 \times 3)$ & 15 & $100 \%$ \\
\hline $1 /(3 \times 4)$ & 15 & $100 \%$ \\
\hline $1 /(4 \times 4)$ & 15 & $100 \%$ \\
\hline $1 /(4 \times 5)$ & 15 & $100 \%$ \\
\hline $1 /(5 \times 5)$ & 15 & $100 \%$ \\
\hline $1 /(5 \times 6)$ & 15 & $100 \%$ \\
\hline $1 /(6 \times 6)$ & 15 & $100 \%$ \\
\hline $1 /(6 \times 7)$ & 14 & $93 \%$ \\
\hline $1 /(7 \times 7)$ & 12 & $80 \%$ \\
\hline $1 /(7 \times 8)$ & 5 & $33 \%$ \\
\hline $1 /(8 \times 8)$ & 1 & $7 \%$ \\
\hline
\end{tabular}

genesis to image processing," IEEE Trans. Image Processing, vol. 5, no. 7, pp. 1137-1150, July 1996.

[3] K. Ito, T. Aoki, and T. Higuchi, "Digital reaction-diffusion system - A foundation of bio-inspired texture image processing —," IEICE Trans. Fundamentals, Vol. E84-A, No. 8, pp. 1909-1918, Aug. 2001.

[4] K. Takita, T. Aoki, Y. Sasaki, T. Higuchi and K. Kobayashi, "High-accuracy image registration based on phase-only correlation and its experimental evaluation," Proc. of 2002 IEEE International Symposium on Intelligent Signal Processing and Communication Systems, pp. 86-90, Nov. 2002.

[5] http://www.higuchi.ecei.tohoku.ac.jp/poc/

[6] J. D. Murray, "Mathematical Biology," Springer-Verlag, Berlin, 1993. 